\title{
High-flux Hydrogen Irradiation-induced Cracking of Tungsten Reproduced by Low-flux Plasma Exposure
}

\author{
L. Gao*, A. Manhard, W. Jacob, U. von Toussaint, M. Balden and K. Schmid \\ Max-Planck-Institut für Plasmaphysik, Boltzmannstr. 2, 85748 Garching, Germany
}

\begin{abstract}
Hydrogen-induced cracking (HiC) or blistering is a commonly observed feature in plasmaloaded material surfaces. HiC exhibits a strong dependence on the irradiation parameters, such as incident flux and fluence, particle energy, and sample temperature. However, the underlying physics of this process is still not understood. Focusing on $\mathrm{HiC}$ with intra-granular cavities in tungsten (W) exposed to deuterium (D) plasma, we apply a 1-dimensional flux-balance model and further propose the crucial role of the solute $\mathrm{D}$ distribution in the subsurface region for initiating $\mathrm{HiC}$ formation in plasma-loaded surfaces. Along this proposal, HiC features previously observed only under high-flux $\left(\sim 10^{24} \mathrm{D} \cdot \mathrm{m}^{-2} \mathrm{~s}^{-1}\right)$, elevated-temperature $(\sim 500 \mathrm{~K})$ exposure conditions - the co-existence of protrusions with intra-granular cavities and small-sized, dome-shaped blisters with inter-granular cracking at the surfaces - were reproduced in our low-flux experiments $\left(\sim 10^{20} \mathrm{D} \cdot \mathrm{m}^{-2} \mathrm{~s}^{-1}\right)$ by loading $\mathrm{W}$ samples at low sample temperatures $(230 \mathrm{~K})$. The presence of protrusions in low-flux experiments is attributed to the comparable local solute $\mathrm{D}$ distribution in the corresponding blistering-relevant depth in both types of D plasma exposure. Applying the 1-dimensional flux-balance model in interpreting $\mathrm{HiC}$ formation in plasma-loaded surfaces, the present work allows us to further explore the underlying physics of $\mathrm{HiC}$ formation under well-defined experimental conditions.
\end{abstract}

PACS: 52.40.Hf; 52.77.Dq; 68.37.-d; 28.52.Fa;

Key words: Plasma-Surface Interaction; Hydrogen-induced Cracking; Deuterium; Tungsten; Solute distribution.

\begin{tabular}{|c|c|}
\hline $\begin{array}{l}\text { Published in: } \\
\text { doi: }\end{array}$ & $\begin{array}{l}\text { Nuclear Fusion, 59, } 056023 \text { (8pp) (2019) } \\
\text { 10.1088/1741-4326/ab0915 }\end{array}$ \\
\hline Submitted: & 15.01.2019 \\
\hline Accepted: & 21.02 .2019 \\
\hline Available online: & 11.04 .2019 \\
\hline
\end{tabular}

${ }^{*}$ Corresponding author. Fax: +49-3299-1212, Tel: +49-3299-1417.

E-mail address: liang.gao@ipp.mpg.de 


\section{Introduction}

Hydrogen-induced cracking (HiC) or blistering in metals, creating planar cavities (voids or cracks) along grain boundaries or crystallographic lattice planes [1] and often visible as blisters in the surface, is an important issue in many technological applications. In particular for thermonuclear fusion devices, HiC in plasma-facing materials (PFM) is known to affect $\mathrm{H}$ isotope retention, especially the radioactive fuel tritium is here of relevance [2]. Furthermore, it will lead to enhanced erosion and/or dust formation [3], and also degradation of the materials properties [4], resulting in great concerns with respect to safety and cost efficiency [5]. Tungsten (W) is presently the PFM foreseen for the divertor region of ITER [6], which triggered a lot of research work dedicated to $\mathrm{H}$ isotope retention in W. Generally speaking, the interaction of hydrogen with $\mathrm{W}$ and the consequences in terms of $\mathrm{HiC}$ morphologies and hydrogen retention are rather complex. Many aspects of this phenomenon are still poorly understood in spite of a huge amount of research work in the past (see Ref. [5, 7-9] and references therein). For example, it has been widely recognized that $\mathrm{HiC}$ features in plasma-loaded $\mathrm{W}$ surfaces depend strongly on exposure parameters, e.g., ion energy $E_{\text {ion }}$ [10], ion flux $\Gamma_{\text {incident }}[11-13]$, hydrogen isotope fluence $\Phi[11,14]$, and sample temperature $T[15,16]$, etc. However, a fundamental understanding of the underlying physics of this strong dependence on experimental parameters is still missing. Aiming at this, the task is to compare the results from experiments conducted in different devices, which is, however, rather complex to be fulfilled due to their vastly variable exposure conditions. Taking the particle flux $\boldsymbol{\Gamma}_{\text {incident }}$ for $\mathrm{HiC}$ study as an example, it ranges from $10^{18}$ to $10^{24} \mathrm{~m}^{-2} \mathrm{~s}^{-1}$ in the variety of different plasma or ion/atom-beam devices applied for studying $\mathrm{HiC}$ of $\mathrm{W}[11,14,17-22]$. The extremely high particle flux $\left(\geq 10^{24} \mathrm{~m}^{-2} \mathrm{~s}^{-1}\right)$ is investigated because $\mathrm{W}$ materials in the divertor region will have to withstand similar intense bombardment from energetic hydrogen isotopes. The notable exceptions where $\mathrm{W}$ samples were actually exposed to particle fluxes in this range in laboratory experiments did show new blistering features [11, 2022], which, however, are presently not fully understood. A typical drawback for many laboratory plasma devices is that while they allow experiments in a well-controlled environment, they cannot reach the extreme particle fluxes occurring in a divertor of a future fusion device. A similar dilemma applies also to other experimental parameters. Instead of comparing widely varying experimental results from different devices to achieve a physical understanding of $\mathrm{HiC}$ in $\mathrm{W}$, an alternative is to reproduce the observed different blistering features in experiments from one identical device by a controlled variation of selected exposure parameters. This brings the intention of the present work: after deriving a hypothesis of the parameter dependence of $\mathrm{HiC}$ in plasma-exposed $\mathrm{W}$ based on a 1-dimensional flux-balance model [23, 24], we will provide experimental evidence supporting this hypothesis by reproducing in our experiments blistering features so far only observed in high-flux devices with vastly different loading conditions.

\section{Theory}

As a generally-accepted feature in most simulations [25-29] and experimental studies [1, 10-22], a relatively high local hydrogen concentration (higher than some critical value) is required to initiate HiC formation at potential defects under a certain loading condition. In other words, irrelevant of specific loading parameters, W material - provided the same material grade is used and the same mechanical properties apply -is supposed to show similar blistering features if the local solute hydrogen distributions in the relevant depth range are comparable. Therefore, crucial in reproducing HiC features at different loading conditions is to create a comparable solute D distribution in the blistering-relevant depth. Note that, since most metallic materials exhibit a ductile-to-brittle-transition temperature (DBTT), the mechanical properties of pristine $\mathrm{W}$ samples, even if they are from the same production batch, may differ significantly at different temperatures applied during plasma loading. To this end, the temperatures of $\mathrm{W}$ samples selected for comparing the $\mathrm{HiC}$ features after exposure in the present work are always below the reported DBTT of W material [30]. 


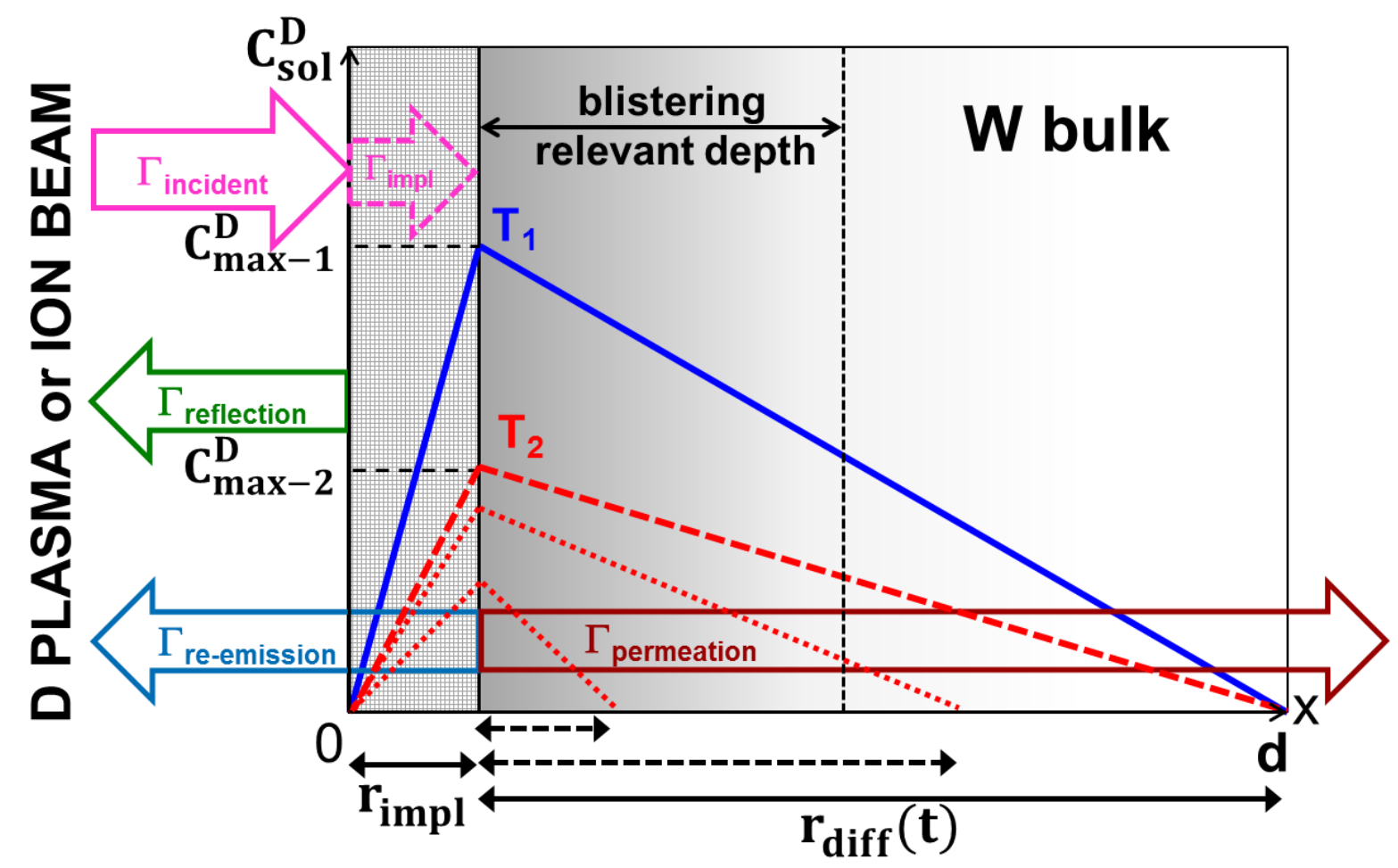

Fig. 1: Schematic of W sample exposed to D plasma. The horizontal axis stands for the depth in the sample while the vertical axis indicates the solute D concentration. The implantation depth $\left(r_{\text {impl }}\right)$ is typically much smaller compared with the blistering-relevant depth (indicated by the vertical dashed line) and also far smaller than the sample thickness $d$. It is assumed that the solute hydrogen concentration is zero at the front and back surfaces (i.e., diffusion-limited boundary conditions) and reaches its maximum $\left(C_{\max }^{D}\right)$ at the implantation depth $\left(\boldsymbol{r}_{\text {impl }}\right)$. The fluxes shown in the scheme are balanced at steady state: $\Gamma_{\text {incident }}-\Gamma_{\text {reflection }}=\Gamma_{\text {reemission }}+\Gamma_{\text {permeation }}$ and thereby building up a linear distribution of the solute hydrogen (shown by solid line at $T_{1}$ and dashed line at $T_{2}\left(T_{1}<T_{2}\right)$. The dotted lines represent the evolution of the solute hydrogen profile at the start of implantation.

Let us now consider an analytic model [23, 24] sketched in Fig. 1: It shows a schematic representation of the solute concentration profile as a function of depth in $\mathrm{W}$ samples during deuterium (D) irradiation. $d$ is the sample thickness and $r_{i m p l}$ the mean implantation depth. D ions are implanted into the sample and come to rest at $r_{i m p l}$. From there they diffuse to both sides and give - in steady state - rise to a linear D concentration profile (e.g., the solid line at temperature $T_{1}$ and the dashed line at $T_{2}\left(T_{1}<T_{2}\right)$ decaying from $r_{i m p l}$ to both surfaces according to Fick's first law. The concentrations at both surfaces are zero if assuming diffusion-limited boundary conditions. Based on Ref. [23, 24] and our own experience, this assumption is often justified for hydrogen plasma exposure of W. Since $r_{i m p} \ll d$, the built-up of the linear profile (e.g., shown by the dotted lines) from $r_{\text {impl }}$ to the implantation surface is normally much faster than that to the rear surface. The $\mathrm{D}$ diffusion front propagates into the sample depth with a so-called 'absorbing boundary' [24] due to trapping by defects in the material until all the trap sites are filled in the corresponding depth. Note that a possible evolution of defects during D implantation is not taken into account. In steady state all fluxes through the whole sample thickness are balanced: $\Gamma_{\text {incident }}-\Gamma_{\text {reflection }}=\Gamma_{\text {reemission }}+\Gamma_{\text {permeation }}$. Both the maximum solute D concentration $\left(C_{\max }^{D}\right)$ at $r_{\text {impl }}$ and 
the concentration gradients depend strongly on the diffusion coefficient determined by the sample temperature. A higher temperature will enhance diffusion thereby reducing $C_{\max }^{D}$ and the concentration gradients to the surfaces. Additionally, $C_{\max }^{D}$ depends on $r_{i m p l}$ determined by the impinging ion energy since a larger $r_{i m p l}$ will reduce the out-diffusion to the nearby implantation surface. Furthermore, particles with higher impinging energies will have lower reflection yields, which will in turn increase $\Gamma_{i m p l}$ for a given $\Gamma_{\text {incident }}$ (i.e., $\Gamma_{\text {impl }}=\Gamma_{\text {incident }}-\Gamma_{\text {reflection }}$ ). For realistic experimental conditions we have $r_{i m p l} \square d$ and in turn $\Gamma_{\text {reemission }} \gg \Gamma_{\text {permeation, which means }} \Gamma_{\text {reemission }} \approx \Gamma_{\text {impl }}$ this leads to an expression for $C_{\max }^{D}$ at steady-state:

$$
C_{\max }^{D}=\frac{r_{\text {impl }} \times \Gamma_{\text {reemission }}}{\rho_{W} \times D(T)}
$$

where $\rho_{W}$ is the atomic density of $\mathrm{W}$ and $D(T)$ the diffusion coefficient at temperature $T$. For exposures with the same ion energy, both $r_{i m p l}$, and the reflection yield, are constant, and as a consequence, $C_{\max }^{D}$ remains a function of $\Gamma_{\text {incident }}$ and $T$. By adjusting $T$, one should be able to achieve comparable $C_{\max }^{D}$ and in turn comparable solute $\mathrm{D}$ distributions in the near-surface region even with very different $\Gamma_{\text {incident }}$. Therefore, a way to reproduce HiC in $\mathrm{W}$ observed in high-flux plasma exposures at elevated temperatures in a low-flux experiment is to work at low temperatures. Following this idea, in the present work, we exposed $\mathrm{W}$ samples in a low-flux plasma device $\left(\sim 10^{20} \mathrm{D} / \mathrm{m}^{2} \cdot \mathrm{s}\right)$ at very low sample temperature $(230 \mathrm{~K})$ and successfully reproduced $\mathrm{HiC}$ features previously only found after high-flux $\left(\sim 10^{24} \mathrm{D} / \mathrm{m}^{2} \cdot \mathrm{s}\right)$ plasma exposures at elevated temperatures [11-14].

\section{Experimental}

Poly-crystalline, hot-rolled W samples $\left(15 \times 12 \times 0.8 \mathrm{~mm}^{3}, 99.97\right.$ wt. \% purity, Plansee SE, Austria), with grain sizes of the order of $1 \mu \mathrm{m}$ [31], were chemo-mechanically polished to a mirror-like finish and annealed in vacuum at $1200 \mathrm{~K}$ for 2 hours. D implantations were performed in the quantified plasma source 'PlaQ' [32] delivering primarily $\mathrm{D}_{3}{ }^{+}$ions (94\%) with minor contributions of $\mathrm{D}_{2}{ }^{+}(3 \%)$ and $\mathrm{D}^{+}$ ( $3 \%$ ). The applied ion bombardment energy of $\sim 115 \mathrm{eV}$ corresponds to a mean energy of $38 \mathrm{eV}$ per $\mathrm{D}$ for the dominant ion species $\mathrm{D}_{3}{ }^{+}$, which is chosen to be identical to the deuteron energy used in the high-flux experiments [11] for comparison. The used D flux was $1 \times 10^{20} \mathrm{D} \cdot \mathrm{m}^{-2} \mathrm{~s}^{-1}$ and the accumulated $\mathrm{D}$ fluence was $6 \times 10^{24} \mathrm{D} \cdot \mathrm{m}^{-2}$. The samples were mounted with screws on a tungsten-coated copper holder. The biased sample holder is electrically isolated from grounded cooling structure using an aluminium nitride disc, which has a very high heat conductivity of $180 \pm 10 \mathrm{Wm}^{-1} \mathrm{~K}^{-1}$. Temperature control is achieved by two external fluid thermostats connected to two separate cooling channels. The separate cooling channels allow access to the combined temperature range covered by the thermostats without replacing the heat transfer medium first. Both thermostats are feedback-controlled by a thermocouple pressed against the sample holder from the back side. A closed-circuit thermostat with high-temperature oil as the heat transfer medium is used for temperatures between about 320 and $600 \mathrm{~K}$. A low-temperature thermostat using ethanol as the heat transfer medium is technically able to produce temperatures down to $180 \mathrm{~K}$ and can be used up to about $310 \mathrm{~K}$ for safety reasons. It was used for the work presented here to maintain a sample temperature of $230 \pm 5 \mathrm{~K}$ during plasma exposure. Morphological modifications at the surface and in the sub-surface of the samples after $\mathrm{D}$ exposure were investigated by scanning electron microscopy (SEM) assisted by focused ion beam (FIB) cross-sectioning. 


\section{Results and discussion}

Typical HiC features observed in W surfaces exposed to high-flux D plasmas are the co-existence of the 'classical' dome-shaped blisters with cavities at grain boundaries [11-14] and the 'special' irregularshaped protrusions [11] with in-grain cavities (see Figs. $2 \& 5$ in Ref. [11] and Figs. 1 \& 2 in Ref. [14]). So far such protrusions have not been observed in $\mathrm{W}$ at comparable depth (i.e., $\sim 200 \mathrm{~nm}$ ) after low-flux exposure at temperatures of $300 \mathrm{~K}$ [18] or higher [19, 33] with otherwise comparable exposure parameters. Following the hypothesis above that the local solute D distribution determines if HiC will occur or not, we propose that the required local solute $\mathrm{D}$ distribution for the formation of protrusions is too high to be reached in the relevant depth in low-flux plasma exposure at temperatures above $300 \mathrm{~K}$.

Fig. 2 shows the surface modification and sub-surface cracking features of $\mathrm{HiC}$ in $\mathrm{W}$ after low-flux exposure at $230 \mathrm{~K}$ with a fluence of $6 \times 10^{24} \mathrm{D} / \mathrm{m}^{2}$. An overview image in Fig. 2a and details of features probed by scanning electron microscope (SEM) (Figs. 2b-f) are compiled. A careful comparison shows that almost identical blistering features as found on the high-flux exposed W surfaces [11-14] are produced here by low-flux exposure at $230 \mathrm{~K}$. Some small-sized, dome-shaped blisters with diameters up to $5 \mu \mathrm{m}$ (Fig. 2c) and a lot of small, irregular-shaped nanostructures $(<300 \mathrm{~nm})$ formed within individual grains become visible at higher magnification (Figs. $2 \mathrm{~b} \& \mathrm{c}$ ). Both types of blistering features are in areal density and size distribution very similar to those found for the high-flux exposure (see, e.g., Fig. 5 in Ref. [11] and Fig. 2 in Ref. [14]). Further investigations with FIB cross-sectioning (Figs. 2 d-f) also show almost identical cracking features: all the protrusions investigated here show intra-granular cracking and the corresponding cavities are at comparable depths within isolated grains. Note that the $\mathrm{W}$ material grades used in both experiments might slightly differ. However, because both W samples were produced by the same company and in both experiments the sample temperatures are below the reported DBTT for $\mathrm{W}$ [30], it is reasonable to assume comparable mechanical properties with respect to blistering formation at these two conditions. The incident ion flux reported in Ref. [11] is more than 4 orders of magnitude higher $\left(\sim 10^{24} \mathrm{D} \cdot \mathrm{m}^{-2} \mathrm{~s}^{-1}\right)$ than the $\mathrm{D}$ flux used in the experiment described here and the sample temperature was $\sim 500 \mathrm{~K}$ [11]. Using Frauenfelder's diffusion coefficient of hydrogen in W [34], i.e., $D(T)=D_{0} \cdot \exp \left[-0.39 /\left(k_{B} T\right)\right]$ with $D_{0}=2.9 \times 10^{-7} \mathrm{~m}^{2} \mathrm{~s}^{-1}$ (Frauenfelder's value for $\mathrm{H}$ divided by $\sqrt{2}$ ), yields a diffusion coefficient which is more than 4 orders of magnitude lower at $230 \mathrm{~K}$ than at $500 \mathrm{~K}$ $\left(D_{230 K}=8.47 \times 10^{-16} \mathrm{~m}^{2} / \mathrm{s}\right.$ and $D_{500 K}=3.48 \times 10^{-11} \mathrm{~m}^{2} / \mathrm{s}$, respectively $)$. The reflection yield is estimated ( $\sim 0.70$ for deuteron with $38 \mathrm{eV}$ on $\mathrm{W}$ surfaces) based on Eckstein's calculation for light projectiles [35]. $\Gamma_{\text {incident }}$ in Ref. [11] is $\sim 2 \times 10^{24} \mathrm{D} \cdot \mathrm{m}^{-2} \mathrm{~s}^{-1}$ while the flux used in this work is $\sim 1 \times 10^{19} \mathrm{D} \cdot \mathrm{m}^{-2} \mathrm{~s}^{-1}$ [32]. As a result, a roughly 4-times higher $C_{\max }^{D}$ was achieved in the present low-flux experiments $\left(1.4 \times 10^{-3} \mathrm{D} / \mathrm{W}\right)$ compared with that in the high-flux experiments $\left(3.5 \times 10^{-4} \mathrm{D} / \mathrm{W}\right)$, which finally gives rise to a similar solute $\mathrm{D}$ distribution in the blistering-relevant depth and accordingly to similar blistering features. Note that, Fig. 1 is not drawn to scale but rather for illustration. E.g., depending on the exposure conditions, the diffusion length is often much larger than the implantation depth and the blister-relevant depth. 


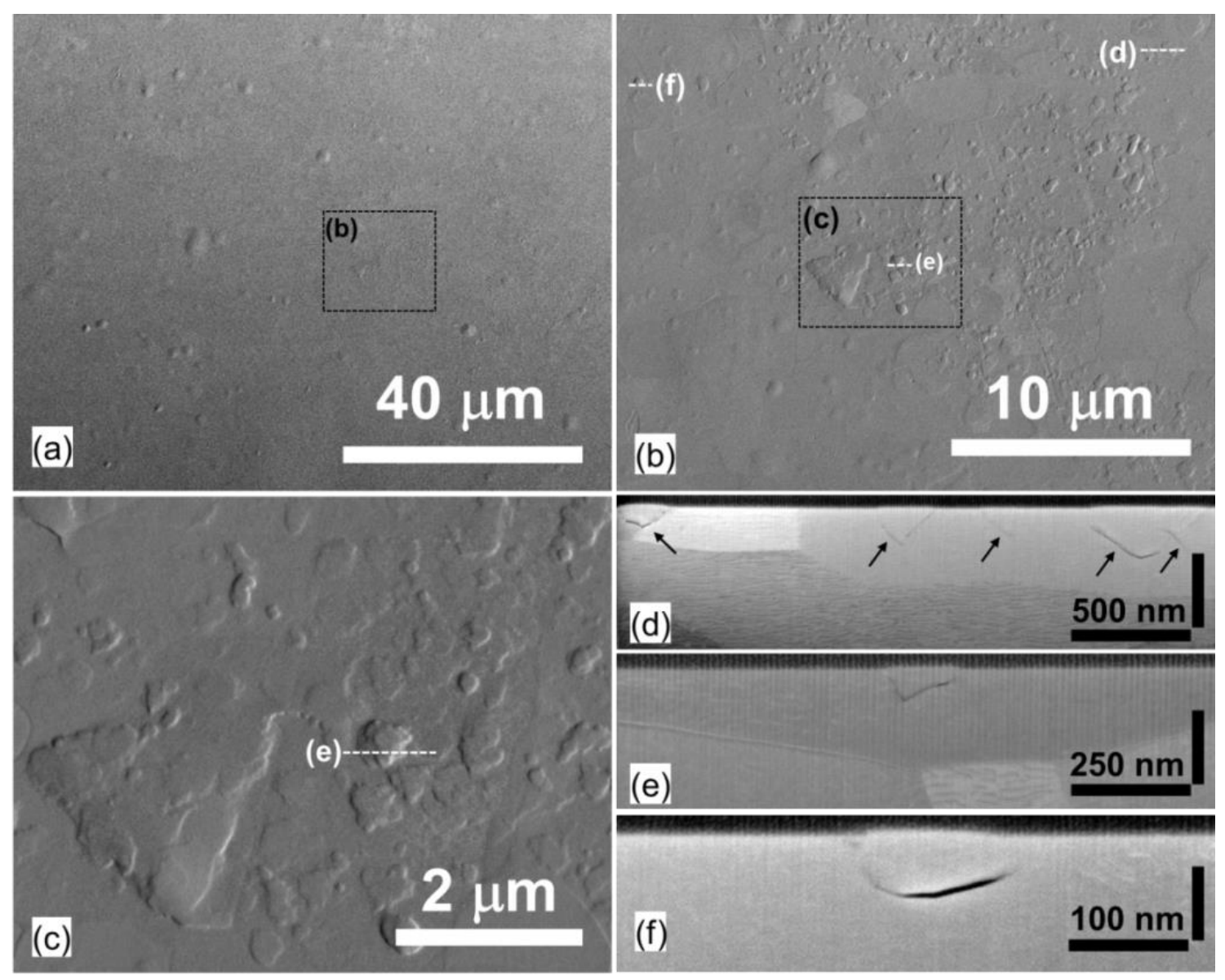

Fig. 2: Surface morphology of W samples exposed to low-flux D plasma at temperature of $230 \mathrm{~K}:$ a)-c) are overview of the surface morphology in different magnification. $d$ )-f) are detailed cross-section images to show the protrusions with intra-granular cracks, some of which are indicated by the arrows in (d).

As a reasonable assumption, we take the deepest point of the cavity as the initiation point of the crack formation. All the presently observed in-grain cracks are initiated in depths $(>100 \mathrm{~nm})$ much larger than the implantation depth (a few $\mathrm{nm}$ at $38 \mathrm{eV}$ per D). Such a fact validates the assumption that blistering is not caused due to direct implantation of $\mathrm{D}$, i.e. at $r_{i m p l}$, but due to $\mathrm{D}$ diffusion and oversaturation in the relevant depth. This is actually the basic assumption to apply the 1-dimentional flux-balance model for interpreting $\mathrm{HiC}$ formation, i.e., using the here-derived $C_{\max }^{D}$ to describe the local solute $\mathrm{D}$ distribution responsible for the blistering features. It should be pointed out that the linear concentration profile drawn in Fig. 1 is the upper limit of the solute D concentration in steady state. Realistically, this steady state could be very difficult to reach especially for very low temperatures as presented here, so the actual solute D concentration should be lower at a certain depth. Nevertheless, this will not hamper our attempt to take $C_{\max }^{D}$ as a good empirical criterion to judge if $\mathrm{HiC}$ with a certain feature (i.e., intra-granular cavities for the present work) forms during plasma exposure. It should be further pointed out that the correlation between the depth of crack nucleation and $C_{\max }^{D}$ requires additional information on the material properties (e.g., defect distribution and their interaction with the solute hydrogen). This is beyond the scope of this work and will hopefully be clarified in the near future. 


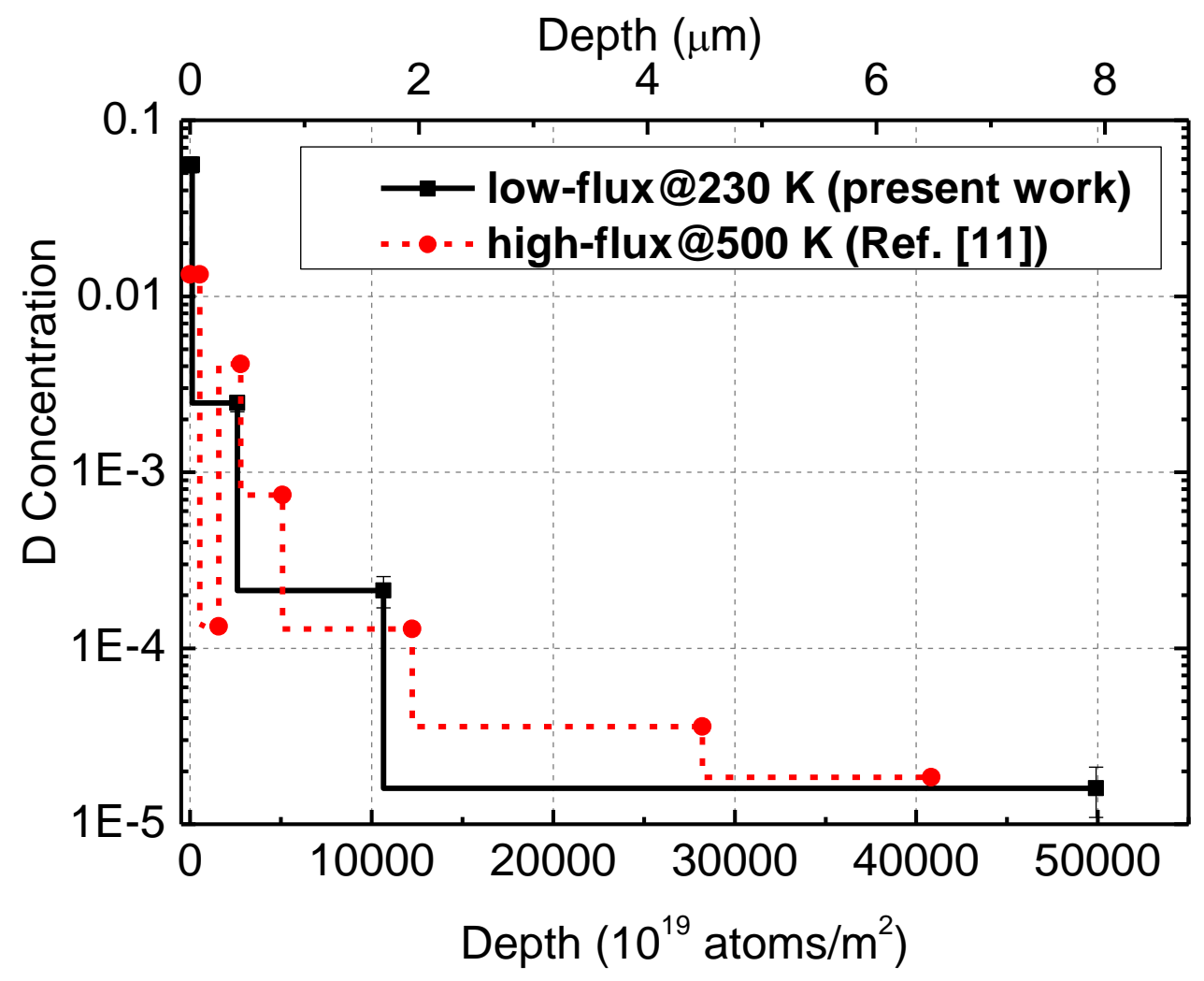

Fig. 3: D depth profiles in $W$ samples exposed to low-flux D plasma at temperature of $230 \mathrm{~K}$ (solid line) and high-flux experiments at $500 \mathrm{~K}$ (dashed line). The high-flux data is re-drawn from Ref. [11], where the applied D fluence $\left(10^{26} \mathrm{D} \cdot \mathrm{m}^{-2}\right)$ is 2 orders of magnitude higher than that of the low-flux exposure from the present work $\left(6 \times 10^{24} \mathrm{D} \cdot \mathrm{m}^{-2}\right)$

The depth profile of D retained in the low-flux-exposed W sample measured after loading is compared with that from high-flux exposure [11], as shown in Fig. 3. Considering, on the first glance, the very different experimental conditions, the two depth profiles in Fig. 3 are strikingly similar. However, as mentioned above, we specifically compare these experiments because they are characterized by similar $C_{\max }^{D}$ values. We regard this as further evidence that the local solute concentration profile, which we describe by $C_{\max }^{D}$, is indeed a key value for comparing plasma exposure experiments. It should be mentioned that the applied D fluence $\left(10^{26} \mathrm{D} \cdot \mathrm{m}^{-2}\right)$ is more than one order of magnitude higher in Ref. [11] than in the low-flux exposure of the present work $\left(6 \times 10^{24} \mathrm{D} \cdot \mathrm{m}^{-2}\right)$. The observation that the $\mathrm{D}$ retention depth profiles for these two cases are comparable may indicate that they are influenced by blisters and that the blisters have formed already at relatively low fluence. It has been shown earlier that blisters or protrusions will enhance reemission of $\mathrm{D}$ after implantation and in turn reduce the diffusion flux into larger depth [36, 37]. This means the additional D fluence after the formation of such blistering features will not significantly modify the D retention profile beyond the cavities.

In addition, knowing that $\mathrm{W}$ samples exposed to identical conditions as applied in the here presented experiments but at $300 \mathrm{~K}$ do not show protrusions [11, 18], we determined the critical temperature for initiating protrusions by varying the temperature from $230 \mathrm{~K}$ to $300 \mathrm{~K}$ with a fixed ion energy (i.e., $38 \mathrm{eV}$ per D). The D fluence for determining the critical temperature for the appearance of protrusions is set to $6 \times 10^{22} \mathrm{D} / \mathrm{m}^{2}$. This low fluence is already sufficient to distinguish whether protrusions appear. SEM imaging of each sample after implantation was performed to check if protrusions appear or not. It is confirmed that no protrusion appear for temperatures higher than $280 \pm 5 \mathrm{~K}$. Taking Eq. 1 and also the 
required constants from Ref. $[34,35]$, we arrive at a $C^{D}{ }_{\max }$ of $1.0 \times 10^{-4} \mathrm{D} / \mathrm{W}$, which is a factor of 3 lower than the $C^{D}{ }_{\text {max }}$ value reached in in Ref. [11]. Extrapolating this critical $C^{D}{ }_{\text {max }}$ determined from the present low-flux experiments to high-flux exposure yields a higher temperature limit (i.e., higher than $500 \mathrm{~K}$ ), above which protrusions do not appear on $\mathrm{W}$ surfaces under comparable exposure condition as in Ref. [11], which is roughly $700 \mathrm{~K}$. From literature [15, 38, 39], where the used W materials were all from PLANSEE, we indeed find that no protrusions are found in $\mathrm{W}$ after irradiation at a temperature above $710 \mathrm{~K}$ with similar flux as in [11], which fits to our prediction quite nicely. It should be noted that this high temperature threshold already exceeds the DBTT and accordingly modifies the mechanical properties of W. Within the present framework, we cannot predict in how far this change will affect HiC formation during D exposure.

One may argue that the gas pressure in equilibrium $p_{e q}$ with $\mathrm{D}$ in solution should be the driving force for bubble growth by hydrogen precipitation [40,41]. Due to the large endothermic enthalpy of solution for $\mathrm{H}$ in tungsten (1.04 eV/atom [34]), $p_{e q}$ is much higher at low temperature than at high temperature for a given concentration of $\mathrm{D}$ in solution. If pressure alone would be the dominating factor then a significant difference in $p_{e q}$ between blistering and non-blistering conditions would be expected. To test our proposal that $C^{D}{ }_{\max }$ is crucial for determining if protrusions occur in $\mathrm{W}$ surfaces after $\mathrm{D}$ plasma loading, we made a broad survey in literature about the occurrence of similar HiC features (i.e., intra-granular cracking) in comparable polycrystalline $\mathrm{W}$ material grades. We calculate all the $C^{D}{ }_{\max }$ and the corresponding $p_{\text {eq }}$ for different flux-temperature combinations from literature as well as from the present work, as shown in Figs. 4 and 5, respectively.

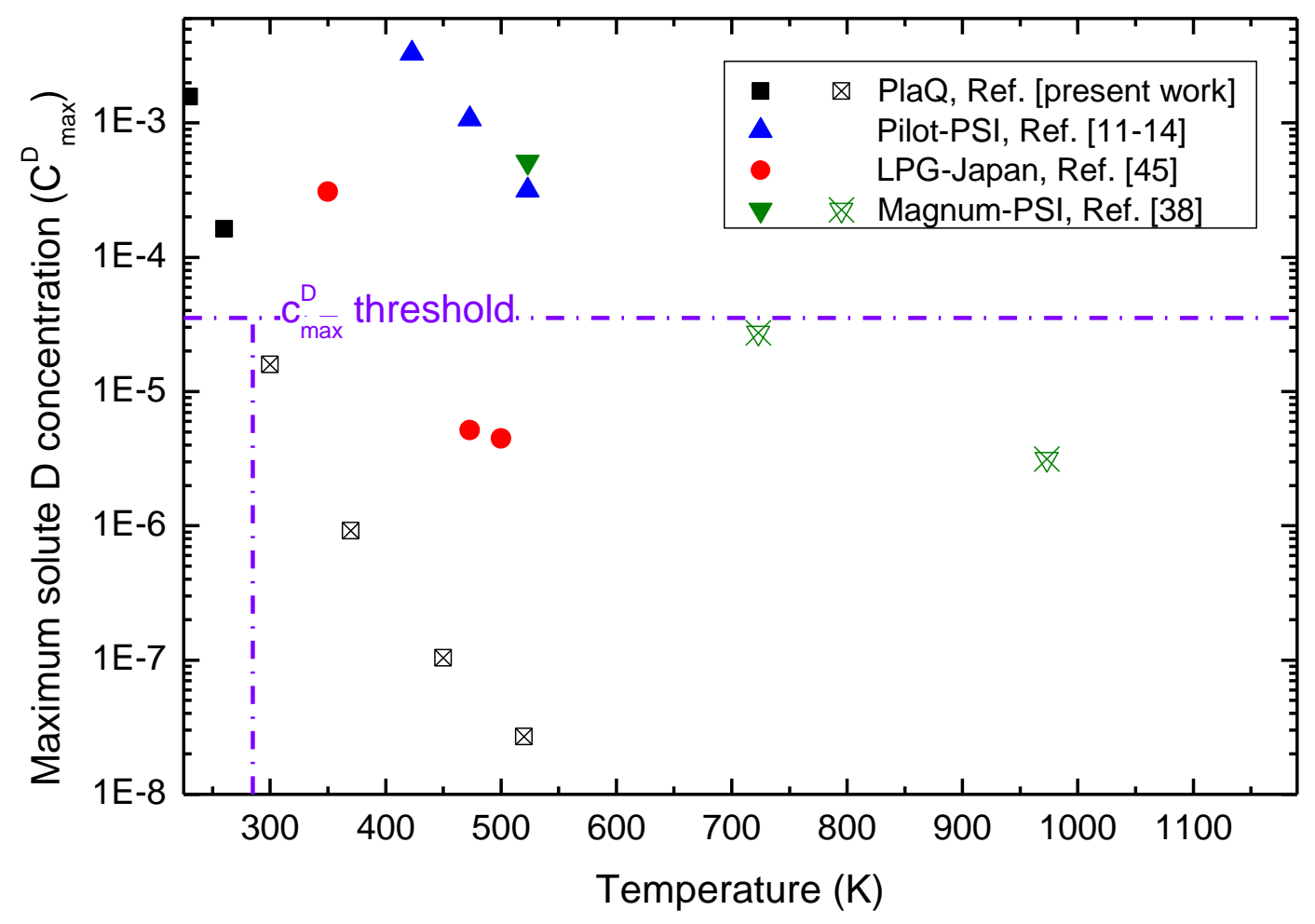

Fig. 4: The maximum solute $D$ concentration $C^{D}{ }_{\text {max }}$ under different flux-temperature combinations from different devices collected from literature to determine the occurrence of protrusions (i.e., intra-granular cracking) in plasma-loaded $W$ materials. Filled symbols correspond to protrusions occurring and open symbols means conditions without protrusions occurring. The dash-dotted line indicates the critical $C^{D}{ }_{\text {max }}$ extrapolated from lowflux plasma devices (i.e., $285 \mathrm{~K}$ in PlaQ) in the present work. Most of the W materials selected for comparison are polycrystalline $W$ from PLANSEE, only the symbols shown as down triangles (i.e., Magnum-PSI) are for polycrystalline W from A.L.M.T. Corp., Japan. 
As one can see in Fig.4, it seems that the whole dataset can be distinguished in terms of protrusions occurrence by the critical $C^{D}{ }_{\text {max }}$ determined in the present work (i.e., the dash-dotted line), except for the two data set shown as red circles. Two data points should according to our threshold not to show blistering. They are actually from exactly the same material as for the present work but exposed to much higher fluence in LPG device [42]. This may indicate an additional effect of the exposure fluence on the occurrence of protrusions, particularly near the threshold conditions. We will address this issue in a future experiment. It should be stressed that for the comparison in Fig. 4, we only selected literature data where the used $\mathrm{W}$ material is comparable to that in our study, and the protrusion features are very similar to those in our experiments and in [11]. As illustrated in, e.g. [19], the response of W during plasma loading depends also strongly on material grades. Indeed, some literature results do not follow the proposed threshold behavior (e.g., [43, 44]), but these were obtained using different $\mathrm{W}$ grades and/or showed different surface features. We, therefore, plan to extend our investigations also to other $\mathrm{W}$ grades and possibly other metals in the future.

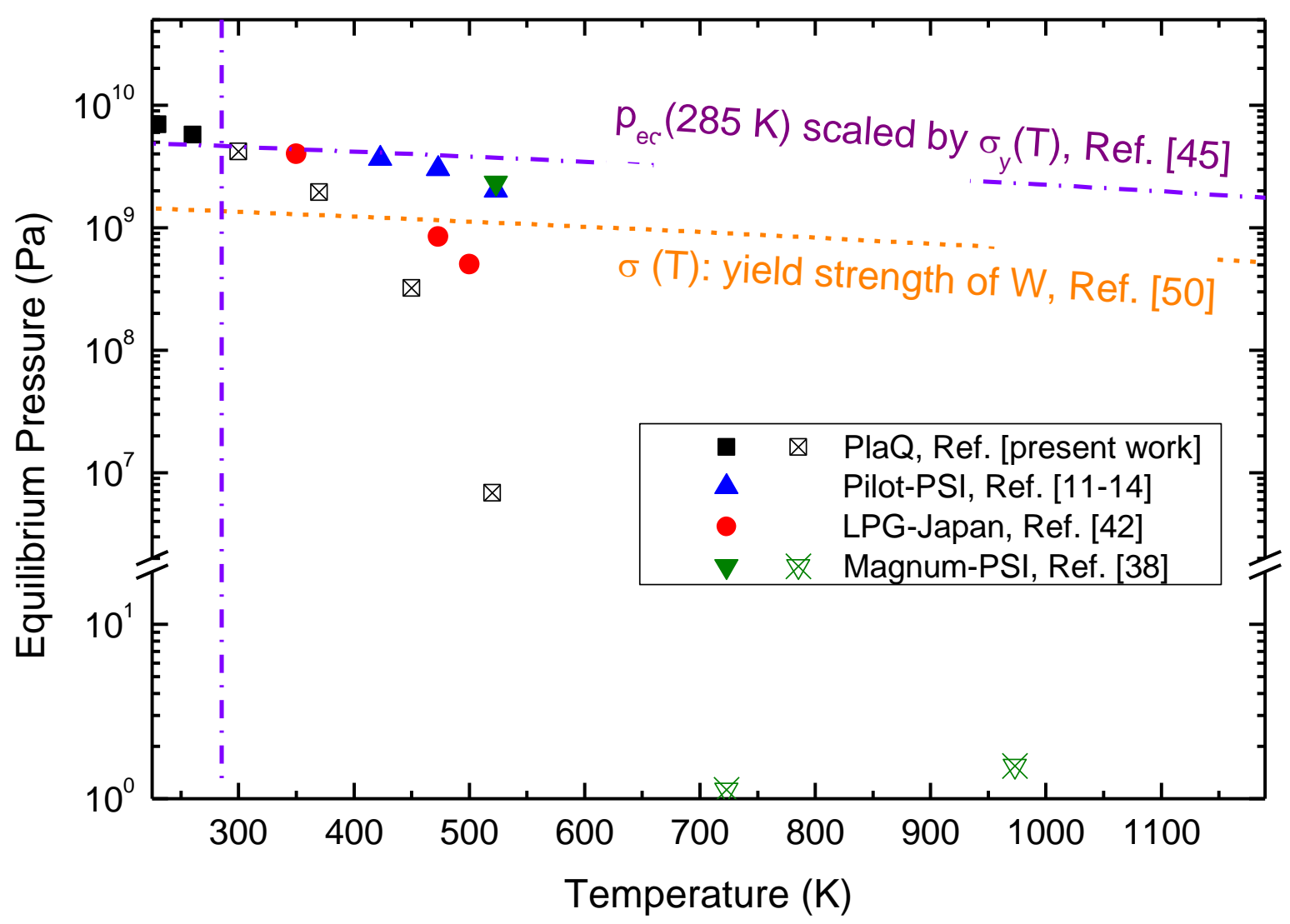

Fig. 5: The corresponding equilibrium pressure $p_{\text {eq }}$ calculated based on Eq.(8) from Ref. [45] for all the $C_{\text {max }}^{D}$ and temperatures in Fig.4. Filled symbols correspond to protrusions occurring and open symbols means conditions without protrusions occurring. The dashed line indicates the yield strength of stress-relieved $W \sigma_{y}$ as a function of temperature [50]. Taken the same scaling factor as for $\sigma_{y}$ for a possible temperature dependence of the critical $p_{\text {eq }}$ the dash-dotted line represents the scaled $p_{\text {eq }}$ calculated based on the critical $C^{D}{ }_{\text {max }}$ acquired from low-flux plasma devices (i.e., $285 \mathrm{~K}$ in PlaQ) in the present work. Except for the data set from the present work, all other data sets do not follow if we take $p_{\text {eq }}$ to judge the occurrence of blistering. Most of the selected $W$ materials for comparison are polycrystalline $W$ from PLANSEE, only the symbols in down triangles (i.e., Magnum-PSI data points) are for polycrystalline W from A.L.M.T. Corp., Japan [39]. 
Similar comparisons on the equilibrium pressure $p_{e q}$ for the same dataset to judge if protrusions occur or not are shown in Fig.5. The $p_{e q}$ is calculated based on the extrapolation of the presently-determined $C^{D}{ }_{\max }$ threshold but following the equation provided in Ref. [45] (Eq. 8). While our empirical criterion for protrusion occurrence with respect to $C^{D}{ }_{\text {max }}$ in Fig. 4 has some limitations with respect to the material grade, it nevertheless appears to be a better threshold condition than $p_{e q}$ in Fig. 5 in the context of the present work. By this, we would rather rule out that the deciding process for the occurrence of the observed HiC features is bubble growth due to pressure [41, 42]. Instead, we propose that the important step is actually the nucleation of the bubbles. According to our findings, these processes should be closely related to the solute D concentration. Possible candidates would be superabundant vacancy formation [46, 47], which could facilitate the nucleation of vacancy clusters, or hydrogen-enhanced de-cohesion [48, 49 and references therein], which would facilitate $\mathrm{HiC}$ nucleation at crystal defects. These processes are affected by the material microstructure: presence of grain boundaries, vacancy clusters and dislocations, either intrinsic or from radiation damage. This is in line with the finding that the critical solute concentration for $\mathrm{HiC}$ is different for different $\mathrm{W}$ material grades.

\section{Conclusion}

In summary, the solute D distribution in the near surface region is proposed to play a crucial role on blister formation in plasma-exposed or ion-irradiated surfaces. This proposal is corroborated by qualitative conclusions drawn from a 1-dimensional flux-balance model. By working at low-temperature $(230 \mathrm{~K})$, we reproduce in low-flux experiments the near-surface solute $\mathrm{D}$ concentration profile and in turn $\mathrm{W}$ blistering features with intra-granular cavities observed otherwise only after high-flux exposures. This work provides a basic framework to understand the underlying physics of $\mathrm{HiC}$ formation in plasma-loaded surfaces, i.e., taking $C_{\max }^{D}$ as an empirical criterion to decide which HiC morphologies could be expected for the chosen $\mathrm{W}$ grades. Applying this framework allows, on the one hand, a comparison of experiments performed under vastly varying experimental conditions, and, on the other hand, enables further studies by producing different $\mathrm{W}$ blistering features in laboratory plasma device with high reproducibility and good control of the relevant parameters.

\section{Acknowledgements}

We are grateful to Gabriele Matern for help on sample preparation. Thanks are further due to Michael Fußeder and Joachim Dorner for help with the NRA measurements. This work has been carried out within the framework of the EUROfusion Consortium and has received funding from the Euratom research and training program 2014-2018 and 2019-2020 under grant agreement No 633053. Work performed under EUROfusion WP PFC. The views and opinions expressed herein do not necessarily reflect those of the European Commission.

\section{References:}

[1] Condon J. B. and Schober T. 1993 Hydrogen bubbles in metals J. Nucl. Mater. 2071

[2] Causey R. A. 2002 Hydrogen isotope retention and recycling in fusion reactor plasma-facing components J. Nucl. Mater. 30091

[3] Shu W. M., Wakai E. and Yamanishi T. 2007 Blister bursting and deuterium bursting release from tungsten exposed to high fluences of high flux and low energy deuterium plasma Nucl. Fusion 47201

[4] Yao W. Z., Wang P., Manhard A., Krill Iii C. E. and You J. H. 2013 Effect of hydrogen on the slip resistance of tungsten single crystals Materials Science and Engineering: A 559467

[5] Roth J. et al. Tritium inventory in ITER plasma-facing materials and tritium removal procedures. Plasma Phys Contr F 50, 103001 (2008). 
[6] Pitts R. A. et al. 2013 A full tungsten divertor for ITER: Physics issues and design status J. Nucl. Mater. 438, Supplement $\mathrm{S} 48$

[7] Myers S. M. et al. 1992 Hydrogen interactions with defects in crystalline solids Rev. Mod. Phys. 64559

[8] Pundt A. and Kirchheim R. 2006 Hydrogen in metals: Microstructural Aspects Ann. Rev. Mater. Res. 36555

[9] Heinola K., Ahlgren T., Nordlund K. and Keinonen J. 2010 Hydrogen interaction with point defects in tungsten Phys. Rev. B 82094102

[10] Luo G. N., Shu W. M. and Nishi M. 2005 Incident energy dependence of blistering at tungsten irradiated by low energy high flux deuterium plasma beams J. Nucl. Mater. 347111

[11] Hoen M. H. J. t., Balden M., Manhard A., Mayer M., Elgeti S., Kleyn A. W. and Emmichoven P. A. Z. v. 2014 Surface morphology and deuterium retention of tungsten after low- and high-flux deuterium plasma exposure Nucl. Fusion 54083014

[12] Buzi L., Temmerman G. D., Unterberg B., Reinhart M., Litnovsky A., Philipps V., Oost G. V. and Möller S. 2014 Influence of particle flux density and temperature on surface modifications of tungsten and deuterium retention J. Nucl. Mater. 455316

[13] Zayachuk Y., Manhard A., 't Hoen M. H. J., Jacob W., Zeijlmans van Emmichoven P. A. and Van Oost G. 2015 The effect of ion flux on plasma-induced modification and deuterium retention in tungsten and tungstentantalum alloys J. Nucl. Mater. 46469

[14] Xu H. Y., Liu W., Luo G. N., Yuan Y., Jia Y. Z., Fu B. Q. and De Temmerman G. 2016 Blistering on tungsten surface exposed to high flux deuterium plasma J. Nucl. Mater. 47151

[15] Shu W. M., Isobe K. and Yamanishi T. 2008 Temperature dependence of blistering and deuterium retention in tungsten exposed to high-flux and low-energy deuterium plasma Fusion Eng. Des. 831044

[16] Alimov V. K., Shu W. M., Roth J., Lindig S., Balden M., Isobe K. and Yamanishi T. 2011 Temperature dependence of surface topography and deuterium retention in tungsten exposed to low-energy, high-flux D plasma J. Nucl. Mater. 417572

[17] Markelj S., Založnik A., Schwarz-Selinger T., Ogorodnikova O. V., Vavpetič P., Pelicon P. and Čadež I. 2016 In situ NRA study of hydrogen isotope exchange in self-ion damaged tungsten exposed to neutral atoms $J$. Nucl. Mater. 469133

[18] Manhard A. 2012 Deuterium inventory in tungsten after plasma exposure: A microstructural survey. PhD thesis, Univ. Augsberg, IPP Report 17/34, Max-Planck-Institute für Plasmaphysik

[19] Balden M., Manhard A. and Elgeti S. 2014 Deuterium retention and morphological modifications of the surface in five grades of tungsten after deuterium plasma exposure J. Nucl. Mater. 452248

[20] Xu H. Y., Luo G. N., Schut H., Yuan Y., Fu B. Q., Godfrey A., Liu W. and Temmerman G. D. 2014 Enhanced modification of tungsten surface by nanostructure formation during high flux deuterium plasma exposure $J$. Nucl. Mater. 44722

[21] Xu H. Y., De Temmerman G., Luo G. N., Jia Y. Z., Yuan Y., Fu B. Q., Godfrey A. and Liu W. 2015 Deuterium-induced nanostructure formation on tungsten exposed to high-flux plasma J. Nucl. Mater. 463308

[22] Jia Y. Z., Liu W., Xu B., Qu S. L., Shi L. Q. and Morgan T. W. 2017 Subsurface deuterium bubble formation in $\mathrm{W}$ due to low-energy high flux deuterium plasma exposure Nucl. Fusion 57034003

[23] Tanabe T., Furuyama Y., Saitoh N. and Imoto S. 1987 Determination of Deuterium Diffusion Coefficient in Metals by Ion Driven Permeation Technique Trans. Jap. Inst. Metals 28706

[24] Schmid K. 2016 Diffusion-trapping modelling of hydrogen recycling in tungsten under ELM-like heat loads Phys. Scripta. T167 014025. Note: unfortunately, the equation for the diffusion length in the $3^{\text {rd }}$ paragraph of page 6 is supposed to be $\frac{\Delta R_{D}(t)}{\Delta \mathrm{t}}=\frac{\Gamma^{\text {Bulk }}}{C^{\text {Trap }}(T) \rho}$ instead of $R_{D}(\mathrm{t})=\frac{\Gamma^{B u l k}}{C^{T r s p}(T) \rho} t$.

[25] Pressouyre, G. M. \& Bernstein, I. M. 1981 An example of the effect of hydrogen trapping on hydrogen embrittlement. Metall. Trans. A 12, 835.

[26] Liu Y.-L., Zhang Y., Zhou H.-B., Lu G.-H., Liu F. and Luo G. N. 2009 Vacancy trapping mechanism for hydrogen bubble formation in metal Phys. Rev. B 79172103

[27] Sang C., Sun J., Bonnin X., Liu S. and Wang D. 2013 Numerical simulation of the bubble growth due to hydrogen isotopes inventory processes in plasma-irradiated tungsten J. Nucl. Mater. $\mathbf{4 4 3} 403$

[28] Terentyev D., Dubinko V., Bakaev A., Zayachuk Y., Renterghem W. V. and Grigorev P. 2014 Dislocations mediate hydrogen retention in tungsten Nucl. Fusion 54042004 
[29] Lu S., Shuo J., Hong-Bo Z., Ying Z., Wenqing Z., Ueda Y., Lee H. T. and Guang-Hong L. 2014 Critical concentration for hydrogen bubble formation in metals Journal of Physics: Condensed Matter 26395402

[30] D. Rupp, S.M. Weygand. 2011 Loading rate dependence of the fracture toughness of polycrystalline tungsten, J. Nucl. Mater. 417477

[31] Manhard, A., Matern, G. and Balden, M. 2013 A step-by-step analysis of the polishing process for tungsten specimens. Practice Metallography 50, 5-16

[32] Manhard A., Schwarz-Selinger T. and Jacob W. 2011 Quantification of the deuterium ion fluxes from a plasma source Plasma Sources Sci. T. 20015010 Note: Unfortunately, the information given in the last paragraph of this article is not correct, but the information in figures 5 and 6 is correct. The contribution of the molecular ions to the total ion flux for standard conditions is: $\mathrm{D}_{3}{ }^{+}=94 \%, \mathrm{D}_{2}{ }^{+}=3 \%$ and $\mathrm{D}^{+}=3 \%$. Correspondingly, the contributions to the total deuteron flux in form of ions are: $97 \%, 2 \%$, and $1 \%$.

[33] Manhard A., Schmid K., Balden M. and Jacob W. 2011 Influence of the microstructure on the deuterium retention in tungsten J. Nucl. Mater. 415 S632

[34] Frauenfelder R. 1969 Solution and Diffusion of Hydrogen in Tungsten J. Vac. Sci. Technol. 6388

[35] Eckstein, W. 2002 Calculated Sputtering, Reflection and Range Values. Max-Planck-Institut für Plasmaphysik, IPP Report 9/132 (Online link: http://hdl.handle.net/11858/00-001M-0000-0027-4522-5)

[36] Gao L., von Toussaint U., Jacob W., Balden M. and Manhard A. 2014 Suppression of hydrogen-induced blistering of tungsten by pre-irradiation at low temperature Nucl. Fusion 54122003

[37] Bauer J., Schwarz-Selinger T., Schmid K., Balden M., Manhard A. and Toussaint U. v. 2017 Influence of nearsurface blisters on deuterium transport in tungsten Nucl. Fusion 57086015

[38] Unpublished SEM investigations by M. Balden on samples used in the following article: Lee H. T., De Temmerman G., Gao L., Schwarz-Selinger T., Meisl G., Höschen T. and Ueda Y. 2015 Deuterium retention in tungsten exposed to mixed D+N plasma at divertor relevant fluxes in Magnum-PSI J. Nucl. Mater. 463974

[39] Lindig S., Balden M., Alimov V. K., Manhard A., Höschen C., Höschen T., Tyburska-Püschel B. and Roth J. 2011 Sub-surface structures of ITER-grade W (Japan) and re-crystallized W after ITER-similar low-energy and high-flux D plasma loadings Phys. Scripta. T145 014039

[40] Kolasinski R. D., Cowgill D. F. and Causey R. A. 2011 A continuum-scale model of hydrogen precipitate growth in tungsten plasma-facing materials J. Nucl. Mater. 415 S676

[41] Kolasinski R. D., Cowgill D. F., Donovan D. C., Shimada M. and Wampler W. R. 2013 Mechanisms of gas precipitation in plasma-exposed tungsten J. Nucl. Mater. 438, Supplement S1019

[42] Unpublished data via private communication with M. Balden, Max-Planck-Institut für Plasmaphysik 85748Garching b. München

[43] Buzi L., De Temmerman G., Matveev D., Reinhart M., Schwarz-Selinger T., Rasinski M., Unterberg B., Linsmeier C. and Van Oost G. 2017 Surface modifications and deuterium retention in polycrystalline and single crystal tungsten as a function of particle flux and temperature J. Nucl. Mater. 495211

[44] Buzi L. et al. 2015 Influence of tungsten microstructure and ion flux on deuterium plasma-induced surface modifications and deuterium retention J. Nucl. Mater. $\mathbf{4 6 3} 320$

[45] Tkacz M. and Litwiniuk A. 2002 Useful equations of state of hydrogen and deuterium J. Alloy. Compd. 330-332 89

[46] Fukai Y. 2003 Formation of superabundant vacancies in M-H alloys and some of its consequences: a review $J$. Alloy. Compd. 356-357 263

[47] Kato D., Iwakiri H., Watanabe Y., Morishita K. and Muroga T. 2015 Super-saturated hydrogen effects on radiation damages in tungsten under the high-flux divertor plasma irradiation Nucl. Fusion $\mathbf{5 5} 083019$

[48] Oriani R. A. 1972 A mechanistic theory of hydrogen embrittlement of steels Berichte der Bunsengesellschaft für physikalische Chemie 76848

[49] Song J. and Curtin W. A. 2013 Atomic mechanism and prediction of hydrogen embrittlement in iron Nat Mater 12145

[50] ITER Document No. G 74M 16, ITER Material Properties Handbook (2005). 